Théologiques

Théologiques

\title{
Aspect freudien des rites et de leurs répétitions
}

\section{Guy-Robert St-Arnaud}

Volume 4, numéro 1, mars 1996

Les Rites : céder en résistant

URI : https://id.erudit.org/iderudit/602433ar

DOI : https://doi.org/10.7202/602433ar

Aller au sommaire du numéro

\section{Éditeur(s)}

Faculté de théologie de l'Université de Montréal

\section{ISSN}

1188-7109 (imprimé)

1492-1413 (numérique)

Découvrir la revue

\section{Citer cet article}

St-Arnaud, G.-R. (1996). Aspect freudien des rites et de leurs répétitions.

Théologiques, 4(1), 75-94. https://doi.org/10.7202/602433ar

\section{Résumé de l'article}

La mention de la perspective freudienne concernant les rites et leur dimension religieuse se réduit souvent à la seule caractéristique de névrose obsessionnelle. Or, les textes freudiens suivent une orientation différente fondée sur la découverte de l'inconscient. Ce parcours montre d'abord que l'origine de cette assimilation entre rites et névrose obsessionnelle repose sur un mode particulier de recherche de satisfaction. En revanche, l'hystérie donne lieu à une quête de satisfaction différente qui conduit néanmoins à une autre forme de pratiques rituelles. Trois exemples contemporains indiquent la pertinence de l'analyse freudienne. En accordant une importance particulière au sens des rites, voire même à leur dimension symbolique, Freud opère un double renversement. 
Théologiques 4/1 (1996) 75-94.

\title{
Aspect freudien des rites et de leurs répétitions
}

\author{
Guy-Robert ST-ARNAUD \\ Faculté de théologie \\ Université de Montréal
}

Le temps détruit tout ce qu'il crée, et la fin de toute séquence temporelle est,

pour l'entité qui y est impliquée, une forme de mort. (Aldous HUXLEY)

\section{RÉSUMÉ}

La mention de la perspective freudienne concernant les rites et leur dimension religieuse se réduit souvent à la seule caractéristique de névrose obsessionnelle. Or, les textes freudiens suivent une onientation différente fondée sur la découverte de l'inconscient. Ce parcours montre d'abord que l'origine de cette assimilation entre rites et névrose obsessionnelle repose sur un mode particulier de recherche de satisfaction. En revanche, l'hystérie donne lieu à une quête de satisfaction différente qui conduit néanmoins à une autre forme de pratiques rituelles. Trois exemples contemporains indiquent la pertinence de l'analyse freudienne. En accordant une importance particulière au sens des rites, voire même à leur dimension symbolique, Freud opère un double renversement.

Les rites prennent forme en répétant. Or, le retour à l'identique avive l'écoute du psychanalyste sensible aux détours du refoulement. Les rites révèlent une mise en scène empreinte de retours du refoulé. L'adjonction d'une dimension religieuse aux expressions rituelles suscite aussi le soupçon. Rites et religion conduisent-ils inexorablement au constat freudien d'une névrose obsessionnelle? Telle est la demande donnant lieu à cette écriture. Ce texte sur les rites se divise en cinq sections. 
Une première section convie Freud à s'expliquer sur l'importance et la place des rites au regard de sa conception de la religion comme névrose obsessionnelle. La section suivante envisage une alternative à l'aspect obsessionnel de la religion, soit la névrose hystérique. Cette voie pose le défi d'une autre forme de répétition pour les expressions rituelles. Une troisième section montre l'actualité du point de vue de Freud. Trois indications contemporaines sont présentées. Une quatrième section suit de près le questionnement freudien sur le sens des rites. Un double renversement se trouve mis à jour. La cinquième section précise la nouveauté de ce retournement.

Une synthèse retrace les principaux résultats de ce parcours et dégage quelques conséquences.

\section{La religion comme névrose obsessionnelle universelle}

L'utilisation de la perspective freudienne à propos des rites conduit à un aperçu critique. Les rites semblent voués à un infléchissement névrotique. En tant que religieux, ils tombent sous le couperet de l'Avenir d'une illusion. Cette illusion laisse croire à une protection contre les menaces des dieux, ces puissances souveraines et inconnues.

Pour Freud, les dieux se constituent de traits provenant de la résurgence de la figure paternelle. La mère ayant été pour l'enfant la première protection contre le monde extérieur, le rôle dévolu au père se trouve investi d'une ambivalence particulière. Le protecteur par excellence devient aussi le rival à éliminer. Ce père, ultime défenseur de l'enfant, apparaît aussi comme un intrus s'immisçant dans la relation entre l'enfant et la mère. La crainte que le père inspire pour l'enfant se trouve projetée au firmament des dieux (FREUD, 1989, p. 33).

Par conséquent, "la religion serait la névrose obsessionnelle universelle de l'humanité; comme celle de l'enfant, elle dérive du complexe d'CEdipe, des rapports de l'enfant au père »(FREUD, 1989, p. 61). Aussi, les trois caractéristiques de névrose, d'obsession et d'universalité se trouvent rattachées à la religion et à ses rites.

La religion constitue premièrement un symptôme de type névrotique ${ }^{1}$ par rapport à la psychose et à la perversion. Il s'agit de névrose ${ }^{2}$ car le symptôme du

1 La névrose comprend deux formes : l'obsession et l'hystérie.

2 Bien qu'il soit question de névrose en ce qui a trait à l'évolution des symptômes, Freud n'en souligne pas moins des aspects psychotiques comme le délire et la difficulté d'inscription par rapport à la réalité : 
névrosé porte sur le désir. C'est ainsi que Freud pose la question de l'illusion de la religion, en tant que croyance à des formes de divinités : "Ainsi nous appelons illusion une croyance quand, dans la motivation de celle-ci la réalisation d'un désir est prévalente, ... » (FREUD, 1989, p. 45).

La deuxième caractéristique souligne le caractère obsessionnel d'une telle névrose. L'obsession concerne plus précisément la question des rites. En effet, l'obsessionnel se trouve aux prises avec l'élimination de ce qui constitue le soutien ou les fondements mêmes de sa demande. Aussi doit-il tenter de dissimuler toute trace pouvant manifester une atteinte portée au référent à qui s'adressent ses demandes. Les rites obsessionnels s'inscrivent dans cette dynamique du serviteur vouant son maître à un soin inégalé pour mieux en usurper les biens. Comment l'enfant peut-il demander la sécurité à son protecteur alors qu'il souhaite l'élimination de ce même père privatif? Tout geste susceptible de laisser apparaître une telle perspective d'évincement donne lieu à une réduplication de camouflage protecteur.

Le soupçon s'installe chez l'obsessionnel. Le maître a-t-il eu écho du complot? La seule possibilité d'être rassuré sur le non-savoir du destinataire consisterait à lui demander directement. Puisqu'une telle tentative ne ferait qu'en informer le père, créant à nouveau doute et suspicion, l'obsessionnel répète incessamment l'impossible tentative d'éliminer en dissimulant. Il tente d'effacer inexorablement la trace de ce qui persiste pour se prouver minutieusement que rien d'un tel complot n'est su ou n'a effleuré son esprit. Cette réitération opère à son insu. Elle conduit à des rites tels que se laver les mains quarante fois par jour, vérifier constamment qu'une porte est verrouillée à clé, suivre imperturbablement des règles inédites...

Pour Freud, la religion évite l'accentuation de l'obsession en insérant pleinement dans un symptôme déjà existant, à savoir un encadrement de tites (un rituel ) ou d'interdictions de toutes sortes. Sinon, les rites n'auraient de cesse. Par son rituel, la religion fournit des symptômes, du prêt-à-porter.

Sa technique [ concernant la religion ] consiste à rabaisser la valeur de la vie et à déformer de façon délirante l'image du monde réel, démarches qui ont pour postulat l'intimidation de l'intelligence. $\grave{A}$ ce prix, en fixant de force ses adeptes à un infantilisme psychique et en leur faisant partager un délire collectif, la religion réussit à épargner à quantité d'êtres humains une névrose individuelle, mais c'est à peu près tout (FREUD, 1981, p. 31).

De cette remarque, la pratique analytique rappelle le rapprochement entre certains délires et leurs contenus religieux (voir FREUD, 1985, p. 263-324 et LACAN, 1955-56, p. $71-178$ et 321-331). 
De plus, la religion occupe une place définie en termes de frustration, d'interdiction et de privation (FREUD, 1989, p. 15). Le fait de ne pas satisfaire un instinct est une frustration. Le moyen utilisé pour imposer cette non-satisfaction relève de l'interdiction, tandis que l'état qui en résulte est une privation.

La religion instaure un régime de privation concernant tout excitant en contrepartie d'un gavage de piété. Cette façon dont Freud considère l'utilisation de la religion en Amérique (FREUD, 1989, p. 69) ne fait qu'amplifier la critique. Une telle religion maintient dans l'infantilisme. La détresse humaine assigne aux dieux la tâche d'exorciser les forces de la nature, d'assurer la réconciliation de l'humain avec son destin d'être pour la mort, et finalement de compenser les souffrances et les privations que la vie en commun impose aux humains (FREUD, 1989, p. 25). Cette protection ne devient efficace qu'au prix d'un artefact religieux de rituels qui s'y rattachent.

Finalement, la dernière caractéristique a trait au caractère universel de cette névrose obsessionnelle. Dès lors que cette élaboration repose sur la relation entre l'enfant et le père, elle risque de se retrouver dans plusieurs cas.

Dans un premier bilan, la perspective freudienne présente donc la religion en tant que névrose obsessionnelle universelle. La place occupée par les rites dans la structure obsessionnelle mène à un point névralgique. Il existe une relation d'équivalence ${ }^{3}$ entre rite et névrose obsessionnelle, c'est-à-dire un rapport intrinsèque entre les deux. Mais une objection pourrait être adressée à Freud.

Puisque la plupart des croyants ne se lavent pas les mains quarante fois par jour, est-il justifié d'associer la religion à une névrose obsessionnelle universelle? Or, Freud considère précisément cette question :

... : l'analogie entre la religion et la névrose obsessionnelle se retrouve jusque dans les détails, et bien des particularités et des vicissitudes de la formation des religions ne s'éclairent qu'au jour de cette analogie. En harmonie avec tout ceci est ce fait que le arai croyant se trouve à un haut degré à l'abri du danger de certaines affections névrotiques; l'acceptation de la nérrose universelle le dispense de la tâche de se créer une névrose personnelle (FREUD, 1989, p. 62; nous soulignons).

La religion vient circonscrire le développement d'une névrose personnelle en intégrant le sujet dans une dynamique religieuse obsessionnelle préétablie. Pour comprendre l'articulation du développement de Freud, il importe de préci-

3 Selon la logique formelle, la relation d'équivalence entre deux propositions est vraie si et seulement si les deux propositions sont vraies en même temps, ou sont fausses simultanément. Voir QUINE, 1972, p. 61-66. 
ser l'association entre rite et névrose obsessionnelle. Cetre équivalence peut se résumer de la façon suivante. À considérer le type de satisfaction, il n'y a pas de névrose obsessionnelle sans rite, et réciproquement il n'existe pas de rite sans névrose obsessionnelle.

Au premier abord, ce lien peut laisser entendre que la psychanalyse porte un diagnostic directement à partir d'un symptôme. Par exemple, dès qu'un rite se manifeste, immédiatement un psychanalyste devrait conclure à une névrose obsessionnelle. Ou encore, aussitôt qu'un délire intervient, la personne serait automatiquement classée comme psychotique. Pour Freud, la pratique analytique ne se fonde pas uniquement sur les symptômes. Elle analyse plutôt la structuration psychique à partir de laquelle les symptômes évoluent. Le travail de l'analyse cerne la façon dont la satisfaction est cherchée.

L'équivalence entre rite et névrose obsessionnelle ne repose pas d'abord sur l'observation d'un rite. Elle se fonde sur un « en deça " des termes d'obsession et de rite. Cet "en deça " correspond à la structuration psychique de la quête de satisfaction. Pour l'obsessionnel, l'angoisse touche aux fondements mêmes des demandes du sujet. Il tente d'obtenir satisfaction par réduplication de protection dans sa façon de solliciter. La réciprocité entre névrose obsessionnelle et rite existe parce que obsession et rite établissent un même rapport ${ }^{4}$ avec cette structure de satisfaction.

Au niveau personnel, chaque analysant effectue un travail analytique révélant ce type, ou un autre, de recherche de satisfaction. Telle est la condition pour pouvoir affirmer qu'il s'agit de névrose obsessionnelle. Au niveau universel, à partir de la relation entre le père et l'enfant, Freud retrouve une même structuration de la satisfaction dans la névrose obsessionnelle et les rites. Alors, il l'associe à une source expliquant les religions.

4 Les rapports établis par Freud entre les différents termes peuvent s'écrire formellement :

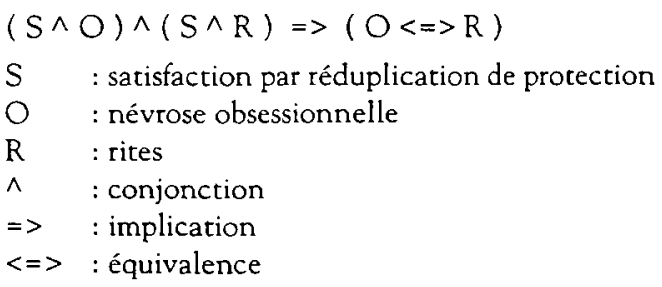

L'énoncé précédent est tautologique, c'est-à-dire qu'il est vrai peu importe la valeur de vérité (vrai ou faux) de chacun des éléments. 
En bref, si la religion favorise et tempère à la fois une névrose obsessionnelle s'exprimant par des formes rituelles, ceci se relie d'abord à la façon dont le sujet religieux met en jeu la recherche de satisfaction. Il reporte à l'extérieur (le monde du divin) la dynamique interne d'ambivalence à l'égard du concurrent et du protecteur (figure paternelle) dont la présence conduit à une privation de l'objet aimé. Pris dans cet engrenage, il en retire pourtant le bénéfice d'une assurance-vie et d'une incessante répétition qui laisse croire à une sécurité.

L'éventualité d'une religion sans rites ne serait-elle pas préférable pour la santé psychique? Mais alors, elle ne correspondrait plus à la même structuration psychique que celle de l'obsession. Par exemple, pourquoi ne pas envisager la religion comme une hystérie, soit l'autre versant de la névrose? Dans l'Avenir d'une illusion, Freud ne semble pas exploiter cette voie. Ailleurs dans Psychologie collective et analyse du moi, il n'aborde pas directement la religion mais bien le christianisme. Cette fois, il est question de névrose hystérique.

\section{La névrose hystérique comme alternative à l'obsession}

Les rites trouvent aisément leur place dans une perspective obsessionnelle parce qu'ils sont assujettis à un mode spécifique de répétition associé à la névrose obsessionnelle. À preuve du développement précédent, Freud ne fait pas état des rites lorsqu'il traite de l'aspect hystérique du christianisme. La lecture de Freud devrait donc s'arrêter ici puisque l'objet de cet article concerne les rites. Dès lors, rites et religions se retrouveraient sous le seul constat d'une névrose obsessionnelle et universelle.

La suite du développement exige un changement de direction. Étant donné que Freud lui-même met en rapport l'hystérie et le christianisme, et que cette religion se constitue de rites, on peut demander comment s'articulerait une structure rituelle selon une dynamique hystérique? La recherche d'une autre voie, pour aborder la religion, conduit-elle à une autre forme de répétition donnant lieu à une expression rituelle différente? La présentation de Freud établissant des liens entre les foules et la névrose hystérique fournit des indications pertinentes.

Afin de situer succinctement la dynamique hystérique, rappelons quelques remarques de Lacan qui retracent le point de départ de Freud :

Elle [ l'hystérique ] veut que l'autre soir un maître, qu'il sache beaucoup de choses, mais tout de même pas qu'il en sache assez pour ne pas croire que c'est elle qui est le prix suprême de tout son savoir. Autrement dit, elle veut un maître sur lequel elle règne. Elle règne, et il ne gouverne pas. 
C'est de là que Freud est parti. Elle, c'est l'hystérique, mais ce n'est pas forcément spécifié à un sexe ${ }^{5}$ (LACAN, 1969-70, p. 150).

La satisfaction liée à la gouverne du maître, l'hystérique y tend au détriment de son désir :

En quête sans répit de ce que c'est qu'être une femme, elle ne peut que tromper son désir, puisque ce désir est le désir de l'autre, faute d'avoir satisfait à l'identification narcissique qui l'eût préparée à satisfaire l'un et l'autre en position d'objet (LACAN, 1966, p. 452; nous soulignons).

et

C'est pour ce qu'elle n'est pas qu'elle entend être désirée en même temps qu'aimée (LACAN, 1966, p. 694; nous soulignons).

Cette quête d'amour de l'hystérique est menée jusqu'à la question de sa propre identité sexuelle : "Suis-je une femme ou un homme?». Ce processus occupe une telle place dans la dynamique hystérique que Freud y reconnaît une source d'identification particulière, soit l'identification hystérique (FREUD, 1976, p. 128-129). Par exemple, une petite fille contracte la toux de sa mère pour manifester qu'elle veut être comme la mère pour en accaparer son " aimant ", le père. L'aspect amoureux se trouve au cœur de la problématique hystérique.

Dans sa présentation de deux foules conventionnelles, Freud montre comment le rapport amoureux instaure une illusion qui rappelle la dimension névrotique :

Dans l'Église (et nous avons tout avantage à prendre pour modèle l'Église catholique) et dans l'Armée, quelques différences qu'elles présentent par ailleurs, règne la même illusion, celle de la présence, visible ou invisible, d'un chef (le Christ dans l'Église catholique, le commandant en chef dans l'Armée) qui aime d'un amour égal tous les membres de la collectivité (FREUD, 1976, p. 113; nous soulignons).

Cette illusion d'un amour égal à chaque membre revêt une importance stratégique puisqu'elle constitue le point névralgique de la cohésion de l'ensemble : « Tout le reste se rattache à cette illusion; si elle disparaissait, l'Armée et l'Église ne tarderaient pas à se désagréger, dans la mesure où le permettrait la contrainte extérieure "(FREUD, 1976, p. 113). Cette croyance en un amour égal marque bien la primauté du désir qui caractérise l'illusion. Freud considère même ce

5 Lacan ne réserve pas l'hystérie à un sexe. Lorsqu'il fait mention d'une femme, il s'agit d'une position subjective, d'une place. 
point comme spécifique au christianisme en tant qu'il vise à une morale plus élevée :

Il [le chrétien] doit, d'une part, s'identifier avec le Christ et, d'autre part, aimer les autres chrétiens comme le Christ les a aimés. [...] On peut être bon chrétien sans jamais avoir l'idée de se mettre à la place du Christ et d'étendre, comme il l'a fait, son amour sur tous les hommes. L'homme faible ne peut pas avoir la prétention de s'élever à la grandeur d'âme et à la force du Christ. Mais c'est en entretenant et en favorisant cette prétention que le christianisme cherchait à obtenir une morale plus élevée (FREUD, 1976, p. 165).

Bien que cette prétention n'ait pas été atteinte, elle n'en continue pas moins à faire fonctionner le christianisme comme cohésion d'un corps. En ce sens, elle marque une problématique du désir orienté vers la question amoureuse, telle que la présente la névrose hystérique.

L'identification hystérique transposée au christianisme comme foule semble trouver quelques rapprochements avec le type d'identification entre les humbles et le Christ. En effet, l'identité du Christ se perd pour se retrouver également en chaque personne sans aucune distinction de sexe:

En ce qui concerne l'amour égal dont le Christ aime tous ses fidèles sans exception et sans distinction, il est nettement exprimé dans ces mots : tout ce que vous faites à l'un quelconque de mes frères les plus humbles, c'est à moi que vous le faites (FREUD, 1976, p. 113; nous soulignons).

La particularité de ce fonctionnement se trouve mise en évidence lorsqu'un processus semblable est reporté à l'armée. Cette fois, le mouvement d'égalité d'amour tourne au ridicule lorsqu'un soldat s'identifie non seulement à ses camarades dans un rapport de réciprocité, mais en plus à son chef : «Vous l'imitez presque dans sa manière de cracher et de s'agiter " (FREUD, 1976, p. 164-5).

Par rapport au christianisme, la différence ne tient pas à l'amour manifesté par le chef d'armée. En effet, le chef d'armée " est le père qui aime également tous ses soldats, et c'est pourquoi ces derniers sont rattachés les uns aux autres par des liens de camaraderie " (FREUD, 1976, p. 114). Toutefois, un soldat n'a pas à devenir chrétien car le militaire n'est pas invité à aimer ses camarades comme son chef les aime, c'est-à-dire en s'identifiant à lui.

Pour la personne chrétienne, une telle identification ne présente rien de surprenant puisqu'il est appelé à aimer comme le Christ a aimé : « Je vous donne un commandement nouveau : aimez-vous les uns les autres. Comme je vous ai 
aimés, aimez-vous les uns les autres » ( Jn 13,34 et 15,12; nous soulignons). Ce rapport entre les individus conduit à un type de répétition qui reste à préciser.

Dans ces deux foules, l'Église et l'armée, Freud note des phénomènes de désagrégation de foule sous l'influence de l'induction ou contagion (FREUD, 1976, p. 117). La perte du lien avec le chef provoque la disparition de ce qui reliait les individus de la foule les uns aux autres. De plus, Freud reprend cette expression de contagion à propos d'une jeune élève de pensionnat qui reçoit une lettre éveillant sa jalousie. Par contagion psychique (FREUD, 1976, p. 129), la crise d'hystérie qui suit la réception de la lettre provoque la même réaction chez quelques-unes de ses amies par identification hystérique.

Ces considérations, orientées à partir de la lecture de Freud du christianısme, ne font état ni de rites, ni de rituels. L'hystérie donne lieu à une modalité de récurrence que Freud nomme induction ou contagion. Une telle façon d'envisager la religion, comme hystérique, oriente l'étude du rite en rapport avec l'instant où le même symptôme se propage à différents sujets. L'aspect hystérique, en tant que mode de diffusion qui comporte une dimension de récurrence, fait du rite un élément circonstancié. Quoi qu'il en soit, l'hystérie révèle une dimension névrotique. L'illusion se perpétue par sa prétention à un amour égal.

\section{Actualité du point de vue de Freud}

Aborder les rites sous le couvert de deux illusions, celle d'une protection et celle d'un amour égal, peut laisser perplexe. L'Avenir d'une illusion et la psychologie collective de Freud rejoignent peut-être le lot des œuvres du passé. D'une part, un mode d'identification, tel celui de l'hystérique, ne disparaît pas subrepticement puisque des personnes de structure hystérique existent encore aujourd'hui. D'autre part, affirmer que le portrait freudien de la religion et de ses rites ne correspond plus au contenu actuel et plus évolué du champ religieux n'enlève pas la pertinence de l'interrogation freudienne sur ce qui a été de l'ordre d'une névrose de l'humanité en sa dimension universelle. Une telle névrose devrait à tout le moins laisser encore quelques traces. Trois indications pourraient corroborer cette perspective.

La première concerne la dimension hystérique qui resurgit sur les lignes téléphoniques de réseau de voyance faisant appel à "l'énergie d'amour». En quête de la solution miracle d'un dieu "tout-amour ", combien de fois n'émerge pas le diagnostic de " carence affective "?

La deuxième indication touche plus spécifiquement la névrose du côté obsessionnel. Dans le champ des nouveaux mouvements religieux, la part importante accordée aux techniques conduisant à l'expérience d'illumination pourrait marquer un retour en force des rites. 
La troisième indication a trait aussi à une utilisation de la religion comme névrose obsessionnelle. Dans ce cas, il s'agit de l'aspect de privation et d'interdiction. Si Freud semble dépassé pour les uns, d'autres contemporains s'y réfèrent pourtant. En 1990, le groupe ENIGMA édite un album de musique et de chanson d'un style non neutre. Laissant à d'autres le soin de se prononcer sur un caractère maléfique ou pas, cette voie d'investigation nous semble inappropriée. Ce groupe est l'instigateur de la chanson intitulée " Mea culpa ». Cette chanson, qui débute sur l'air grégorien d'un kyrie eleison avec un arrière-fond de basses imposant un rythme quasi militaire, a figuré au palmarès des ondes radiophoniques. L'ensemble de l'album laisse peu de place pour n'y voir qu'un jeu de hasard.

La référence à un univers religieux se trouve marquée d'une ambivalence. Le titre "Mea culpa ", associé à la musique grégorienne de caractère dit sacré, avec son contenu "kyrie eleison", contraste vivement avec le rythme des basses qui s'impose au point d'évincer la connotation spirituelle de la musique sacrée. Cette ambivalence ouvre majestueusement le passage ténu d'une douce subversion du fleuron de la spiritualité à l'envoûtement d'une possession amoureuse. Si le grégorien imbu d'un regain de popularité pouvait encore, pour certaines personnes, symboliser l'espace intérieur délimitant en toute limpidité une frontière intangible au religieux, il s'avère que cette chanson a littéralement fait voler en éclat cet espace vital. La limite de ce champ a été outrepassée, tel un interdit intangible dont la valeur ultime ne résiderait qu'à le franchir.

Cette interprétation invite certes à la circonspection. Aussi, faut-il ajouter une citation qui accompagne l'album d'ENIGMA:

The pleasure of satisfying a savage instinct, undomesticated by the ego, is uncomparably much more intense than the one of satisfying a tamed instinct. The reason is becoming the enemy that prevents us from a lot of possibilities of plesaure (ENIGMA, 1990).

Cette citation a pour auteur S. Freud, selon l'indication donnée par le groupe ENIGMA. Elle se trouve dans le texte du Malaise dans la civilisation:

La joie de satisfaire un instinct resté sauvage, non domestiqué par le Moi, est incomparablement plus intense que celle d'assouvir un instinct dompté. Le caractère irrésistible des impulsions perverses, et peut-être l'attrait du fruit défendu en général, trouvent là leur explication économique (FREUD, 1981, p. 24).

La chanson "Mea culpa » tire parti du grégorien en faisant jouer à la religion un rôle d'interdiction qui pose une délimitation. La religion, en tant que 
principe d'interdiction et de privation, continue de produire des effets de sens en suscitant des réactions de transgression ${ }^{6}$. Du sens surgit encore de l'univers religieux dans sa dimension névrotique obsessionnelle, et il y éclôt encore par le biais de l'interdiction.

Cet exemple montre de quelle façon du sens advient d'une dimension névrotique, voire obsessionnelle. Une autre voie de production du sens est-elle possible?

\section{L'alternative du sens, un double renversement opéré par Freud}

Une piste d'investigation des rites se dessine, celle de la question du sens. Le sens des rites peut-il conduire à une démarcation entre rites et névrose? À ce sujet, Freud consacre une étude particulière intitulée Actes obsédants et exercices religieux (FREUD, 1989, p. 81-94). Son apport peut sembler à première vue caduque, voire même obsolète.

Freud ne définit pas directement le rite mais plutôt le « cérémonial ». Le cérémonial névrotique se constitue « d'actions surajoutées ou entravées ou bien rangements, lesquels, à l'occasion des actes de la vie quotidienne, sont exécutés toujours de la même manière ou bien d'une façon qui varie suivant des règles données " (FREUD, 1989, p. 84).

Dans un autre texte sur le tabou de la virginité et sur les cérémonials auxquels il donne lieu, Freud tente de fournir des explications fondées sur des représentations sadiques se reliant à la défloration ou au sang. Toutefois, il mentionne que cette direction n'est pas à surestimer au détriment d'une autre voie qui ne se limite pas au domaine sexuel :

Une deuxième explication se détourne également du domaine sexuel, mais elle a une grande portée dans le domaine général. Elle allègue que le primitif est la proie d'une disposition anxieuse persistante et toujours à l'affût, tout à fait semblable à celle dont nous soutenons l'existence pour les personnes atteintes de névrose d'angoisse dans notre théorie psychanalytique des névroses. Cette disposition anxieuse se révélera plus violemment dans des circonstances qui s'écartent d'une façon ou d'une autre de l'habituel, en apportant quelque chose de nouveau, d'inattendu, d'incompris, d'inquiétant. C'est de là que provient le cérémonial, qui s'étendra largement dans les religions ultérieures, cérémonial lié au début de tout nouvel exercice, au

6 Le milieu publicitaire utilise fréquemment cette opposition. Par exemple, une compagnie informatique fait écho des distractions, suscitées par un ordinateur, au cour d'un groupe de moines orientaux en pleine méditation. 
commencement de toute période temporelle, au premier rejeton de l'homme, de l'animal ou de la plante (FREUD, 1985, p. 70; nous soulignons).

L'importance du cérémonial est mise en lien avec l'angoisse générée par l'inattendu et l'inconnu des moments marquants de l'existence. Aussi, les religions y consacrent une place. Par exemple, les rites de passage associés à la naissance, à la vie de couple, à la mort, ont donné lieu à des mours bien établis. Le catholicisme a vite mesuré leur prépondérance en en faisant des sacrements.

Freud établit des similitudes et des différences entre le cérémonial névrotique et les actes d'un rite religieux. D'abord, il dégage trois ressemblances. La première porte sur la peur advenant suite à une omission des gestes à poser. Un deuxième élément commun concerne la soustraction aux activités quotidiennes. Freud en parle en terme d'isolement complet au sens où le sujet ne doit pas être dérangé durant l'accomplissement du cérémonial ou du rite. La troisième ressemblance concerne le caractère méticuleux de l'exécution. Le moindre écart au cérémonial génère une angoisse dont la reprise fidèle constitue un baume. Aussi, la minutie de l'exécution du cérémonial du névrosé lui donne les traits d'un « acte sacré » (FREUD, 1989, p. 84).

Néanmoins, Freud reconnaît des différences entre le cérémonial et le rite religieux. Les cérémoniaux névrotiques connaissent une grande diversité (des gestes compulsifs comme le lavage de mains, des habitudes aussi anodines que celle de garder les meilleurs morceaux d'un plat pour la fin, etc.). Les exercices religieux, du fait de leur caractère collectif, présentent une plus grande uniformité. Et finalement, Freud termine en insistant sur cette surprenante différence :

...; et surtout cette différence que les petits actes du cérémonial religieux ont un sens et une intention symbolique, tandis que ceux du cérémonial névrotique semblent niais et dénués de sens (FREUD, 1989, p. 86; nous soulignons).

Une des caractéristiques du cérémonial névrotique consiste à paraitre dénué de sens. Même pour l'obsessionnel, qui le réitère pourtant maintes et maintes fois, le sens lui échappe souvent. Alors que les actes sacrés d'un rite religieux sont remplis de sens, les cérémonials des obsessionnels n'en semblent pas pourvus.

Cette différence s'avère surprenante car elle amène à une radicale remise en question de la critique freudienne de la religion. Si une telle démarcation fondée sur le sens s'avère exacte, les attributions de névrose obsessionnelle faites à la religion ne résident que sur une fausse estimation de l'équivalence entre rites religieux et cérémonial névrotique. En effet, les uns sont sensés, et l'autre non. 
Par le biais du sens, les rites religieux trouvent alors une utilité symbolique assurant leur justification particulière?

Or, telle n'est pas la perspective freudienne. Freud ne se faisait que l'écho de l'avis général. La recherche psychanalytique instaure une nouvelle coupure. Les ressemblances et les différences entre champ psychanalytique et champ religieux s'agencent d'une autre façon. Afin d'y parvenir, Freud opère un double renversement de perspective. Ce retournement est double, et non pas deux, car il se rattache logiquement à un même élément dont les conséquences resurgissent sur deux versants. En effet, ce double renversement repose sur la découverte de l'inconscient et le travail analytique qui s'ensuit :

Cependant, c'est justement cette différence [ celle du sens] la plus tranchée entre le cérémonial névrotique et le cérémonial religieux qui disparaît lorsque, grâce à la technique d'investigation psychanalytique, on pénètre assez avant pour comprendre les actes obsédants. Cette investigation permet de mettre radicalement fin à l'apparence d'après laquelle les actes obsédants seraient niais et dénués de sens (FREUD, 1989, p. 86; nous soulignons).

Si Freud concède d'emblée une part de sens au cérémonial religieux, sa pratique de la psychanalyse le place aux antipodes des considérations largement admises du non-sens des cérémonials obsessionnels. Aussi, Freud s'empresse de fournir cinq cas montrant que le cérémonial découle de la vie intime des personnes. Par exemple, une femme notait le numéro de banque de chaque billet avant de s'en séparer. Celle-ci pensait à quitter son mari et doutait du sérieux des intentions d'un prétendant. Alors qu'un jour elle avait besoin d'échanger un billet pour de la petite monnaie, l'homme en question changea le billet tout en promettant de ne pas s'en séparer puisqu'elle l'avait touché. Plusieurs fois encline à lui demander le billet, elle n'osa pas. De toute façon, elle n'aurait pu le reconnaître. Aussi, le soupçon n'étant jamais dissipé, elle se mit à noter le numéro de chaque billet.

7

Cette façon de considérer les rites selon leur utilité en les mettant en référence à des conduites réelles (baptême, mariage, funérailles) fournit une normalisation apparente, qui n'est pas celle de Freud, ni de Lacan :

Retrouvons donc les formules limpides que la mort de Mauss ramène au jour de notre attention; les structures de la société sont symboliques; l'individu en tant qu'il est normal s'en sert pour des conduites réelles; en tant qu'il est psychopathe, il les exprime par des conduites symboliques (LACAN, 1966, p. 132).

Le symbolique n'a-t-il droit de cité qu'à être assimilé à la réalité, comme si les mots de la parole humaine n'ont de place « normale " qu'à servir d'étiquettes aux objets! 
L'exemple précédent décrit un cérémonial névrotique. Or, un lien s'établit entre cérémonial névrotique et sens. De ces détails relégués candidement au hasard des habitudes de vie, le travail analytique dégage une dynamique signifiante inscrite au plus intime de la personne :

On apprend à voir que les actes obsédants ${ }^{8}$ sont, sans exception et dans tous leurs détails, pleins de sens, qu'ils sont au service d'intérêts importants de la personnalité et qu'ils expriment et des événements à influence persistante, et des pensées chargées d'affect de l'individu (FREUD, 1989, p. 86; nous soulignons).

En somme, Freud inverse le rapport entre non-sens et sens du cérémonial névrotique. Avant Freud, les cérémonials névrotiques sont relayés au non-sens tandis qu'après lui ils revêtent une importance inestimée tellement leur sens devient révélateur du vécu de l'individu. Telle est la première facette du double renversement. Ce point ne concerne pas explicitement les rites religieux, mais uniquement la lecture désormais signifiante des cérémonials névrotiques. En quoi ceci peut-il avoir des répercussions sur le champ des idées et des comportements religieux? Le deuxième aspect du renversement concerne cette question.

La religion pouvait prétendre se soustraire à la critique d'une névrose obsessionnelle universelle en opposant le sens des rites religieux au non-sens du cérémonial névrotique. Or, la psychanalyse met en relief le sens des cérémonials névrotiques. Donc, l'apport psychanalytique abolit du même coup cette frontière préservant les rites religieux d'un aspect névrotique.

Avant Freud, les défenseurs de la religion pouvaient évoquer la particularité du sens des rites religieux. Les rites religieux étaient extrinsèques à un aspect obsessionnel. À titre exceptionnel, la pratique de certains individus face aux rites pouvait susciter quelques soupçons de névrose mais sans plus. Après Freud, ce recours à la division entre sens et non-sens ne permet plus de situer les rites religieux dans une zone privilégiée qui enlèverait toute possibilité significative de rapprochement avec le cérémonial névrotique. La lecture freudienne arrête là le deuxième versant du renversement.

8 Si Freud distingue actes obsédants et cérémonial, il affirme qu'il n'existe pas de frontière nette entre les deux puisque le cérémonial se constitue de petits actes. II précise que "le plus souvent, les actes obsédants sont issus d'un cérémonial " (FREUD, 1989, p. 85). Aussi, il propose la conclusion suivante quant au sens contenu dans le cérémonial par rapport aux actes obsédants : "Il en est de même du cérémonial proprement dit... " (FREUD, 1989, p. 89). 
Freud ne va pas jusqu'à rendre la monnaie de sa pièce à la religion en affirmant que les rites religieux n'auraient pas de sens. Une telle conséquence redoublerait le caractère d'illusion, à savoir le côté névrotique.

La barrière du sens séparant rites religieux et cérémonial névrotique étant tombée, le champ religieux se trouve-t-il contraint à un retranchement forcé où la psychanalyse devrait être exclue unilatéralement par principe? Freud continue pourtant le dialogue en examinant l'avènement du sens dans les pratiques répétitives du névrosé et du religieux. L'investigation freudienne conduit à un rapport commun aux deux.

\section{Nouveauté de la perspective freudienne}

Une nouveauté surgit de l'approche freudienne. Il s'agit d'une distinction faisant intervenir des éléments de structure, soit une coupure qui sépare et conjoint en un même lieu les réitérations névrotique et religieuse. Freud dégage une dynamique parallèle entre les cérémonials névrotique et religieux. Il s'interroge sur l'introduction d'une distinction portant sur la recherche du sens.

Si les rites religieux et obsessionnels ont du sens, la façon dont le sens surgit ne s'avère-t-elle pas radicalement différente? Les cérémonials obsessionnels nécessitent le travail analytique pour trouver sens tandis qu'il semble évident dans le cas des rites religieux. Cette différence doit-elle opérer une nouvelle séparation entre cérémonial névrotique et rites religieux? Le texte de Freud permet de répondre à cette interrogation. L'articulation du paragraphe suivant gravite autour du terme inconscient :

C'est une des conditions de l'état pathologique que la personne qui obéit à une compulsion le fasse sans en connaître la signification, au moins la signification principale. Seuls les efforts du traitement psychanalytique pourront lui rendre conscient le sens de l'acte obsédant et par là les mobiles qui l'y poussent. Nous exprimons cet état de choses important en disant que l'acte obsédant sert à manifester des mobiles et des représentations inconscientes. Il semble y avoir là une nouvelle différence d'avec les exercices religieux, mais il faut se rappeler qu'aussi bien le dévot isolé exerce en règle générale le cérémonial religieux sans demander quel en est le sens, tandis que le prêtre et l'investigateur peuvent cependant connaître ce sens, le plus souvent symbolique, du rite. Les mobiles qui poussent impérieusement les croyants aux exercices religieux leur restent cependant à tous inconnus, ou bien sont représentés dans leur conscience par d'autres mobiles mis en avant à leur place (FREUD, 1989, p. 89).

Freud considère ici les deux démarches, psychanalytiques et religieuses, selon trois aspects. Les deux se constituent à partir d'éléments qui produisent du 
sens, les deux fonctionnent selon un non-savoir à propos du sens, et, de ce fait, les deux se trouvent enjointes à l'écart structural instauré par l'inconscient. La découverte de l'inconscient déplace l'accent de la simple question du sens à la présence de sens à l'insu des personnes. Si cette évidence semble claire en ce qui a trait à la cure analyrique, Freud rappelle non seulement que plusieurs croyants ignorent le sens des rites religieux qu'ils pratiquent, mais que tous n'en connaissent pas les mobiles, inconscients il va sans dire.

La nouveauté et l'actualité de la perspective freudienne ne résident plus uniquement en un double renversement. L'inattendu consiste à introduire une coupure qui subsume les deux cérémonials sous le concept d'une supposition de savoir mise au faîte d'un sujet. En effet, les deux comportent une dimension de non-savoir qui pose en contrepartie un lieu où le savoir peut être supposé. C'est de ce lieu supposant le savoir qu'une véritable différence des mobiles entre les pratiques répétitives de chaque personne peut être mise au jour. Ainsi, cette distinction fait effet de séparation, soit une coupure.

Faire marche arrière concernant cette position d'un non-savoir sur le sens ou les mobiles des rites religieux équivaudrait à les réduire à la futilité, au nonsens. Pourtant, Freud identifie clairement dans la religion une place où le savoir peut être supposé relativement au sens des exercices religieux : «le prêtre et l'investigateur peuvent cependant connaître ce sens, le plus souvent symbolique du rite " (FREUD, 1989, p. 89). Quant aux mobiles inconscients des exercices religieux, le travail psychanalytique permet de les mettre à jour.

Les rites tombent alors sous les carégories de l'ambivalence du céder ou résister, et structuralement sous une aporie. D'une part, une des particularités du rite réside dans le fait d'opérer à partir du sens qui est d'abord inconnu ou ignoré par la plupart. Laissant place à l'illusion, son point de départ revêt donc un aspect névrotique. D'autre part, préciser dès le début l'enjeu de telle pratique rituelle n'enlève-t-il pas tout l'engouement du céder ou au contraire n'évoque-til pas l'effroi d'une angoisse de l'étrangeté conduisant à résister? Les rites religieux se trouvent au prise avec une entrée impossible, voire empreinte de névrose. Et pourtant, leur accomplissement vise précisément à apprivoiser l'angoisse qui se trouve à la source même de leur surgissement.

Sans un lieu où le savoir peut être supposé quant aux mobiles des rites, ils demeurent soit névrotique, soit d'un accès paradoxal. Conduit à une telle impasse, une tentative d'esquive semble possible. Les rites religieux auraient un sens symbolique alors que les cérémonials névrotiques en seraient privés. Telle serait peut-être leur inénarrable originalité.

La dimension symbolique conduit-elle à préserver les rites religieux des suspicions psychanalytiques en les soustrayant à l'insipide réitération apparente des 
actes obsédants? Freud reconnaît d'emblée que les rites religieux ont le plus souvent un sens symbolique. Et, la même caractéristique n'échappe pas aux actes obsédants dans leur réalisation de sens :

Ils [ actes obsédants] réalisent ceci de deux manières, en tant que représentation directe ou bien en tant que représentation symbolique; il convient donc de les interpréter soit biographiquement, soit symboliquement (FREUD, 1989, p. 86).

En l'occurrence, la dimension symbolique ne change rien à l'impasse puisqu'elle se retrouve aussi bien dans les rites religieux que le cérémonial névrotique.

\section{Synthèse de ce parcours}

Les rites vont de pair avec la répétition. Ils semblent alors se réduire à un aspect obsessionnel que Freud constate aussi dans la religion. L'obtention de satisfaction par réduplication de protection retrouvée dans la névrose obsessionnelle et les tites entretient l'illusion d'une sécurité. Les rites se trouvent directement liés à la dimension obsessionnelle présente dans la religion selon Freud. Ce rapport revêt une telle place que la religion dispense le croyant de développer sa propre névrose. La religion, prise dans un lien indissociable avec les rites, constitue une névrose obsessionnelle universelle.

Une issue possible au constat freudien d'une obsession universelle consiste à considérer les rites selon une autre forme de répétition. L'analyse freudienne des foules, l'Église et l'armée, conduit à une modalité différente de récurrence où un même symptôme se propage à plusieurs sujets. Le rite est alors un élément circonstancié qui se répète par induction ou contagion, selon les termes utilisés par Freud. Cette alternative à l'obsession comporte aussi un aspect névrotique, à savoir l'illusion d'un amour égal.

L'exemple d'une chanson du groupe ENIGMA montre comment la religion fonctionne encore aujourd'hui comme principe d'interdiction et de privation qui se retrouve dans l'obsession. La dimension névrotique fait surgir du sens de l'univers religieux. Le point de vue freudien ne s'avère donc pas inapproprié.

Le sens des rites semble assurer une spécificité aux rites religieux par rapport au cérémonial névrotique. Les uns seraient sensés et l'autre non. Or, la découverte de l'inconscient met en évidence le sens éminemment intime du cérémonial névrotique. Dès lors, la particularité des rites religieux quant au sens se trouve abolie.

Une autre possibilité de distinction a trait à la façon d'obtenir ce sens. Les rites religieux évoqueraient un sens évident tandis que le névrosé le trouverait 
par le biais du travail analytique. Freud situe les véritables mobiles aussi bien du cérémonial névrotique que des exercices religieux comme étant marqués d'un savoir opérant à l'insu des personnes.

Ignorer ce non-savoir contraint les rites à une entrée impossible. Céder aux rites sans savoir leur sens laisse place à l'illusion. Ils se trouvent pris dans une dynamique névrotique puisqu'ils opèrent à partir du sens inconnu ou ignoré. En revanche, donner le sens des rites au départ ne s'avère pas moins problématique. D'une part, ce sens enlève tout l'engouement de la découverte et du céder sans maitriser. De plus, le sens devrait mettre en lumière l'angoisse que le rite tente d'atténuer, et du coup cette signification conduit à résister. D'autre part, donner un sens apparent ne ferait que masquer les véritables mobiles inconscients et entraînerait de nouveau vers la névrose.

Le sens symbolique des tites religieux ne les soustrait pas au dilemme précédent. Aussi, Freud reconnaît d'emblée cette dimension symbolique aux actes obsédants. Que le sens des rites soit symbolique ou non, il n'échappe pas à un lieu où le savoir est supposé.

Des conséquences de ce parcours se dégagent. La réduction inexorable des rites à la seule névrose obsessionnelle ne s'avère pas justifiée. À tout le moins, ceci ne correspond pas à la perspective freudienne sur les actes obsédants et les exercices religieux. D'ailleurs, le développement de Freud conduit à deux dynamiques de répétition qui donnent lieu à des pratiques rituelles opérant par identification hystérique ou par réduplication de protection. Donc les rites n'occupent pas un seul discours.

Cette relativisation du point de vue freudien n'en diminue pas moins la pertinence. En montrant que les cérémonials névrotiques ont du sens, voire même du sens symbolique, Freud va à l'encontre de l'isolement des rites dans un recoin inatteignable des sciences humaines. Sans préjudice à l'importance et à la valeur du sens qui surgit des pratiques rituelles, le parcours freudien interroge la façon dont les rites produisent des effets de sens. Or, c'est là que le bât blesse à nouveau. Les rites se trouvent dans une double impasse.

D'abord, les rapports entre rites et névrose se trouvent maintenant élargis à l'aspect hystérique, et non plus seulement obsessionnel. D'autre part, les rites sont relayés à une entrée paradoxale, celle du céder en résistant. Elle met en cause l'angoisse éprouvée face à l'inattendu des moments de passage. Et pourtant, cette angoisse se trouve à la source du développement des formes diverses de répétition, dont les rites constituent une modalité. En somme, la relecture des textes freudiens conduit à une nouvelle alternative. Ou bien l'entrée dans une pratique rituelle angoisse davantage les gens, ou bien les rites continuent d'offrir une névrose déjà existante qui semble les rassurer. À l'insu des per- 
sonnes, les pratiques rituelles n'échappent pas aux mobiles inconscients qui les constituent.

L'impasse s'avère-t-elle inéluctable? La perspective freudienne laisse entrevoir une issue possible en considérant le lieu où le savoir sur les mobiles du sens des rites peut être supposé. Comment ce lieu et ce savoir peuvent-ils être mis à jour? Pour Freud, le travail analytique n'est pas exclu. La perspective lacanienne permet de poursuivre et d'affiner le point de vue freudien. En effet, le lieu de savoir quant au sens correspond chez Lacan à l'instigation d'une dynamique de transfert ${ }^{9}$.

Cette considération pose d'emblée une série d'interrogations. Si certains rites ont perdu leur importance, est-ce parce que les personnes n'éprouvent plus d'angoisse face aux moments de transition qui jalonnent leur vie, ou bien parce que les pratiques rituelles leur semblent dénuées de sens, ou bien parce que les rites deviennent des lieux d'initiation secrète éveillant la méfiance et l'angoisse, ou bien parce que la façon dont les rites sont proposés aux personnes n'intègre plus la possibilité d'une quête de savoir sur leur propre existence?

\section{Bibliographie}

ENIGMA

1990. « Mea culpa ». Bande sonore, Canada, Virgin Records. FREUD, S.

1987. Résultats, idées problèmes. Tome II, Paris, PUF.

1976. Essais de psychanalyse. Paris, Payot.

1980. L'interprétation des rêves. Paris, PUF.

1981. Malaise dans la civilisation. Paris, PUF.

1985. La vie sexuelle. Paris, PUF.

1989. L'avenir d'une illusion. Paris, PUF.

LACAN, J.

1955-56. Les Psychoses. Livre III, Paris, Seuil, 1981.

9 L'espace imparti à cet article ne nous a pas permis d'expliciter cette piste d'investigation. 
1966. Écrits. Paris, Seuil.

1969-70. L'envers de la psychanalyse. Livre XVII, Paris, Seuil, 1991. QUINE, W.

1972. Méthodes de logique. Paris, Armand Colin. 